\section{Multistatic Adaptive Pulse Compression}

\author{
SHANNON D. BLUNT, Member, IEEE \\ University of Kansas
}

KARL GERLACH, Fellow, IEEE

U.S. Naval Research Laboratory

A new technique denoted as multistatic adaptive pulse compression (MAPC) is introduced which exploits recent work on adaptive pulse compression (APC) in order to jointly separate and pulse compress the concurrently received return signals from $K$ proximate multistatic radars operating (i.e., transmitting) within the same spectrum. For the return signal from a single pulse of a monostatic radar, APC estimates the particular receive filter for a given range cell in a Bayesian sense reiteratively by employing the matched filter estimates of the surrounding range cell values as a priori knowledge in order to place temporal (i.e., range) nulls at the relative ranges occupied by large targets and thereby suppress range sidelobes to the level of the noise. The MAPC approach generalizes the APC concept by jointly estimating the particular receive filter for each range cell associated with each of several concurrently-received radar return signals occupying the same spectrum. As such, MAPC is found to enable shared-spectrum multistatic operation and is shown to yield substantial performance improvement in the presence of multiple spectrum-sharing radars as compared with both standard matched filters and standard least-squares mismatched filters.

Manuscript received December 28, 2004; revised September 13 and November 7, 2005; released for publication February 3, 2006.

IEEE Log No. T-AES/42/3/884442.

Refereeing of this contribution was handled by E. S. Chornoboy.

This work was supported by the United States Office of Naval Research (ONR 31)

Authors' addresses: S. D. Blunt, Dept. of Electrical Engineering and Computer Systems, University of Kansas, Lawrence, KS 66045 , E-mail: (sdblunt@eecs.ku.edu); K. Gerlach, U.S. Naval Research Laboratory, Washington, D.C. 20375.

0018-9251/06/\$17.00 (c) 2006 IEEE

\section{INTRODUCTION}

The increasing demand for spectrum usage rights by the communications industry coupled with the requirement for wider instantaneous bandwidths for radar applications is creating an ever growing need for more efficient use of the RF spectrum [1]. This is compounded by the fact that future sensing technology (of which radar will play a significant role) has been envisioned as taking the form of sensor networks comprised of several interacting sensors [2]. This paper introduces a multistatic method for adaptive pulse compression as a possible mechanism to enable shared-spectrum multistatic radar. Multistatic system-level topics such as pulse-chasing [3] are not addressed here. The focus of this work is upon the signal processing involved in the separation and subsequent pulse compression of multiple concurrently-received shared-spectrum radar return signals originating from multiple transmitters. Note that the term multistatic, as used here, can refer to either multiple shared-spectrum radars on different (possibly widely separated) platforms or multiple shared-spectrum radars upon the same platform.

In a general sense, the notion of multistatic radar has parallels to multiple-access communications in which the received signals from different "users" are discerned according to either their time of arrival (temporal diversity), their operating frequency (spectral diversity), or their assigned waveform/code (waveform diversity) [4]. Temporal diversity is of limited usefulness for radar applications as the resource management requirements of "time on target" and revisit times over the desired coverage area typically do not provide enough time left over for another (let alone several) radar(s) to operate. The method traditionally used to operationally separate various proximate, concurrently-transmitting radars is for each radar to employ a significantly different portion of the frequency spectrum (i.e., spectral diversity). As such, each radar essentially operates independently thereby effectively ignoring the additional information received bistatically from other radars. However, with the spectrum becoming increasingly crowded and the expectation of future radars to employ even greater instantaneous bandwidth for discrimination/imaging tasks, the opportunity to employ spectral diversity is becoming ever more limited.

Conceptually, shared-spectrum multistatic radars employing waveform diversity can provide greater spectrum utilization, greater area coverage with shorter revisit times, less multi-channel receiver hardware (i.e., for multiple received frequencies), and logistically enable the use of substantially greater instantaneous bandwidths as a result of reduced spectral crowding. Waveform diversity, as defined here, is the use of $K$ identifiably different waveforms 
(i.e., polyphase codes modulated onto the respective transmitted pulses) by $K$ different radars concurrently sharing (transmitting within) the same spectrum such that a particular receiver, given the appropriate processing capability, can separate the resulting received signal into its $K$ constituent radar return signals of interest and subsequently perform the usual target detection, identification, tracking, etc. on each individual signal. Shared-spectrum multistatic radar is somewhat analogous to code-division multiple-access (CDMA) used in communications whereby several users occupy the same spectrum at the same time and each is identified by its distinct transmitted code; albeit communications requires much less sensitivity than is necessary for sufficient radar sensing performance. Waveform diversity in this sense is particularly befitting radar since it is already common practice to transmit a modulated/coded pulse (or waveform) and perform pulse compression upon receive.

For the monostatic radar case, the objective of pulse compression is to obtain the high range resolution of a short pulse, yet with considerably lower peak power, by transmitting a frequency or phase-modulated long pulse [5]. The transmitted modulated long pulse, or waveform, is reflected back to the receiver by scatterers (i.e., targets, clutter) that are illuminated by the radar. The respective delays associated with the reflected signals arriving at the receiver constitute a range profile that is representative of the respective distances from the radar to the scatterers and thereby provides radial location information (as well as potentially enabling identification and discrimination).

The received signal, which can be viewed as resulting from the convolution of the transmitted waveform with the range profile illuminated by the radar, is hence comprised of delayed, attenuated (and perhaps Doppler-shifted) versions of the transmitted waveform. A filter matched to the waveform (the well-known matched filter [5]) can be used to extract the range profile of a range swath from the accompanying noise and is typically followed by a detector (or possibly other intermediate processing stages).

For a solitary "point" target in the presence of white Gaussian noise, the matched filter has been shown [5] to maximize the output signal-to-noise ratio (SNR) of the target. The autocorrelation (or in general the ambiguity surface) of the waveform reveals the inherent ability of the matched filter, for the particular waveform, to extract a single target in the presence of other nearby targets which is measured by the relative range/Doppler sidelobes (autocorrelation values with lag $\neq 0$ for the zero-Doppler case). A target that is aligned with the autocorrelation peak will be masked if another, sufficiently larger target is aligned with a significant range/Doppler sidelobe.

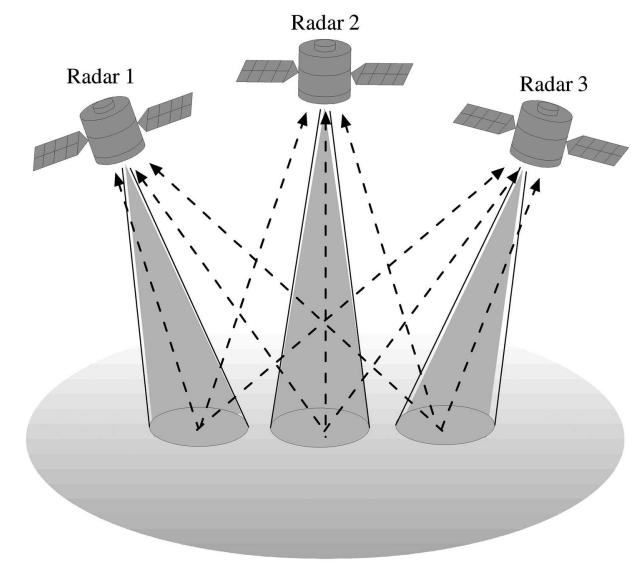

Fig. 1. Example of shared-spectrum multistatic radar configuration.

For a shared-spectrum multistatic radar configuration, we assume that the mainbeam of each of $K$ proximate radars concurrently illuminates one of $K$ different range profiles with a unique waveform (it is assumed that the set of concurrent waveforms have been chosen to possess relatively desirable ambiguity and cross-ambiguity properties yet are not necessarily optimal). Mainbeam footprints may overlap or not. Each individual radar therefore receives the superposition of $K$ signals where the $k$ th signal is comprised of the $k$ th waveform convolved with 1) the range profile illuminated by the mainbeam of the $k$ th radar and 2) the set of range profiles illuminated by sidelobes of the $k$ th radar's antenna pattern, as well as noise. The mainbeam transmit portion of the multistatic radar scenario is illustrated in Fig. 1 for the example of space-based radar. Without loss of generality, at the receiver of radar 1 the estimation of the $K$ distinct range profiles (the $K$ illuminated mainbeam footprints from the perspective of radar 1) therefore involves jointly separating and pulse compressing the set of superimposed received signals associated with the $K$ transmitted waveforms. We point out that the multistatic problem addressed here is quite different from the single-transmitter/multiple-receiver multistatic scenario which has received attention related to synthetic aperture radar (SAR)/ground moving target indication (GMTI) processing (for example [6-8]).

Separation of the received signals associated with the $K$ waveforms can be partially accomplished using beamforming if the $K$ mainbeam footprints have sufficient spatial separation and the radar antenna receive array possesses digital beamforming capability. However, beamforming alone cannot adequately mitigate the mutual interference that is inherent to the shared-spectrum multistatic radar arrangement because it cannot suppress all of the interfering signals present. For the case of no overlap between mainbeam footprints, steering a receive 
beam in the direction of arrival $\theta_{k}$ of the footprint illuminated by the $k$ th radar's mainbeam from the perspective of radar 1 results in a beamformed received signal which contains the desired $k$ th range profile convolved with the $k$ th waveform as well as the other $K-1$ waveforms convolved with their respective composite range profiles and noise. The $i$ th composite range profile (for $i=1,2, \ldots, K$ with $i \neq k$ ) resulting after beamforming in the direction of arrival $\theta_{k}$ is comprised of 1) the range profile illuminated by an antenna sidelobe of the $i$ th radar which overlaps the $k$ th transmit mainbeam footprint and is thereby coaligned with the desired signal in the receive mainbeam, 2) the range profile illuminated by the mainbeam of the $i$ th radar which aligns with a sidelobe of the receive beamformer for the $k$ th signal, and 3) the range profiles illuminated by sidelobes of the $i$ th radar which align with receive sidelobes of the $k$ th beamformer. Because they align with either a transmitter or receiver mainbeam, the first two signals are considered more detrimental to performance than the third set of signals.

To minimize the interference between the received signals, the particular set of waveforms employed for the $K$ multistatic radars must be chosen such as to account somewhat for waveform cross-correlation effects. However, as a result there are fewer available design degrees of freedom for the $K$ waveforms with which to achieve the desired levels of autocorrelation range/Doppler sidelobes. In other words, suppression of cross-correlation interference by means of waveform design comes at the cost of higher autocorrelation sidelobes and vice versa. As such, standard matched filtering, which inherently assumes the presence of a single received signal and noise, will perform poorly in the multistatic scenario due to the combination of increased range/Doppler sidelobes (due to the auto/cross-correlation waveform design trade-off) and mutual interference, both of which effectively cause masking of target returns whenever sufficiently larger target returns are present in any of the superimposed received signals.

Ideally, in order to mitigate the masking problem, a radar's receive filter matched to a particular waveform should, for each particular discrete range cell, also cancel the interference from targets in nearby range cells within the same range profile (i.e., range sidelobes) as well as from target returns from other concurrently received signals (i.e., waveform cross-correlation sidelobes). For the suppression of range sidelobes, least-squares estimation [9-11] has been shown to perform relatively well for the monostatic radar scenario yet it does not account for the presence of multiple received signals and as such can be expected to degrade for the multistatic radar scenario. Hence from a conceptual point of view, it would seem desirable that the receive filter matched to a specific waveform should be adaptive to the actual received signals since the presence of other target returns (from multiple received signals) which may generate masking interference cannot be known a priori and cannot be sufficiently suppressed using standard nonadaptive approaches. As a result of adaptive implementation, each individual range cell associated with each received signal would be estimated using a unique receive filter that suppresses the specific masking interference experienced by the given range cell yet is still closely matched to the desired waveform.

Recently, an adaptive approach based on reiterative minimum mean-square error (RMMSE) estimation known as adaptive pulse compression (APC) was developed for monostatic radar [12-14] and evaluated via simulation. For the monostatic radar case APC is capable of almost completely mitigating range sidelobes thereby enabling estimation of the range profile illuminated by a radar to within the accuracy of the noise. The elimination of range sidelobes is accomplished by adaptively estimating the appropriate receiver pulse compression filter to use for each individual range cell in which the filter estimate is obtained in bootstrapping fashion based upon initial matched filter range cell estimates. Furthermore, APC has been shown to be robust to rather severe Doppler mismatch [13, 14] as well as being superior to standard matched filtering and mismatched filtering via least-squares estimation.

The contribution of this paper is the generalization of the monostatic APC concept to address the problem of multistatic radar reception for which the extraction of useful information is considerably more difficult due to the presence of RF interference from the other $K-1$ radars as well as the necessity to employ waveforms that are nonoptimal (in the autocorrelation sense). The resulting algorithm is denoted as multistatic adaptive pulse compression (MAPC) $[15,16]$. After beamforming, MAPC jointly estimates the appropriate pulse compression filter to use for each individual range cell of the range profile of interest and the $K-1$ interfering composite range profiles. As such, both the range sidelobe interference within the range profile of interest as well as the cross-correlation interference from large target returns in the composite range profiles are suppressed. The implication of such an approach yielding adequate performance is that there is real potential for enabling true multistatic shared-spectrum radar thereby providing a multiplicative improvement in radar spectral efficiency as well as the other possible benefits of either greater area coverage or shorter revisit times. In addition, the MAPC adaptive receive processing is found to compensate somewhat for performance loss that would otherwise occur due to poor waveform selection (such as due to the auto/cross-correlation waveform design trade-off) 
and thus enables much greater freedom in choosing waveforms.

The remainder of the paper is organized as follows. Section II introduces the discrete domain received signal representation for shared-spectrum multistatic radar operation. Section III generalizes the monostatic APC algorithm for the multistatic environment. Some implementation issues are discussed in Section IV. Finally, simulation results are presented in Section V.

\section{RECEIVED SIGNAL REPRESENTATION}

Consider $K$ radars (designated individually as radar $k$, for $k=1,2, \ldots, K$ ) that operate concurrently in the same spectrum each with a distinct transmit waveform (albeit not necessarily selected for optimality in either an auto- or cross-correlation sense). Each radar therefore receives a superposition of the return signals reflected from the different range profiles illuminated by the $K$ transmitters and noise from which it is desired to accurately estimate a portion (denoted as the processing window) of the $K$ mainbeam-illuminated range profiles (or some subset thereof). It is assumed that the radars possess sufficient synchronization and/or exist in an appropriate spatial configuration (e.g. a cluster of space-based radars or a split-aperture radar) such that direct path reception (without reflection) either does not interfere with the received radar return signals or can be adequately rejected. We denote the discrete-time version of the $k$ th radar's waveform as the column vector $\mathbf{s}_{k}$ having length $N$. Assuming an $M$-length linear array at radar 1 (in general MAPC can be applied to any array geometry), we denote $\mathbf{r}_{k}=\left[\begin{array}{ll}1 & e^{j \theta_{k}} \cdots e^{j(M-1) \theta_{k}}\end{array}\right]^{T}$ as the spatial steering vector corresponding to the angle of arrival (AOA) of the reflected return signal resulting from the $k$ th radar's mainbeam illumination which is incident at radar 1 (without loss of generality the same processing is to be performed at each of the radars; thus for their development we consider only radar 1).

Accounting for all of the incident signals at radar 1 , the $\ell$ th time sample on the $m$ th antenna element of radar 1 is defined as

$$
y_{m}(\ell)=\sum_{i=1}^{K}\left[\sum_{k=1}^{K}\left(\mathbf{x}_{i, k}^{T}(\ell) \mathbf{s}_{i} e^{j(m-1) \theta_{k}}\right)+\mathbf{x}_{i, \mathrm{SL}}^{T}(\ell) \mathbf{s}_{i}\right]+v_{m}(\ell)
$$

for $\ell=0, \ldots, L+N-2$ the range indices of the received signal samples of interest where $\mathbf{x}_{i,}(\ell)=$ $\left[x_{i, .}(\ell) x_{i, .}(\ell-1) \cdots x_{i, \cdot}(\ell-N+1)\right]^{T}$ is an $N$-length vector of discrete range profile samples at delay $\ell$ with which the discrete transmitted waveform $\mathbf{s}_{i}$ convolves, and $v_{m}(\ell)$ is additive noise. The term $\mathbf{x}_{i, k}(\ell)$ is the $N$-length portion of the range profile in the mainbeam footprint of the $k$ th radar which is illuminated by either the mainbeam $(i=k)$ or a sidelobe $(i \neq k)$ of the $i$ th radar. The term $\mathbf{x}_{i, \mathrm{SL}}(\ell)$ is an $N$-length portion of the superposition of range profiles illuminated by the sidelobes of the $i$ th radar which do not coincide with a mainbeam footprint illuminated by one of the other $K-1$ radars. The range profiles of interest are those which correspond to mainbeam-transmit/mainbeam-receive $(i=k)$ and the rest are interference to be suppressed. The received radar return signals on the $M$ antenna elements for the $\ell$ th time sample are collected into the vector $\mathbf{y}(\ell)=\left[y_{0}(\ell) y_{1}(\ell) \cdots y_{M-1}(\ell)\right]^{T}$ upon which beamforming is to be performed.

Let each antenna array element possess its own receive channel (frequency down-conversion, A/D converter, I and Q formation, etc....) thus enabling digital beamforming. A separate beamformer is applied across the $M$ outputs of the antenna array for each of the $K$ mainbeam-illuminated regions of interest. At a given radar receiver, for each of the mainbeam range profiles of interest (need not necessarily be all $K$ ), it is assumed that the receiver possesses knowledge of the AOAs of the received radar return signal from the desired mainbeam-illuminated regions. Note that if the receiver only desires to extract an estimate of the range profile which it itself illuminates, then no AOA knowledge of the other radars is needed. Furthermore, as the same processing is performed to estimate each individual mainbeam-illuminated region of interest (whereby the return signals resulting from the other $K-1$ waveforms act as interference), without loss of generality we consider only the estimation of the $n$th range profile (assuming its AOA is known).

Beamforming in the direction of the $n$th mainbeam-illuminated region $(n \in\{1,2, \ldots, K\})$, the $\ell$ th time sample of the resulting signal (after normalization) is denoted as $z_{n}(\ell)$ which is found to be

$$
\begin{aligned}
z_{n}(\ell) & =\frac{1}{M} \hat{\mathbf{r}}_{n}^{H} \mathbf{y}(\ell) \\
& =\sum_{i=1}^{K}\left[\sum_{k=1}^{K}\left(\eta_{n, k} \mathbf{x}_{i, k}^{T}(\ell) \mathbf{s}_{i}\right)+\tilde{\mathbf{x}}_{i, \mathrm{SL}, n}^{T}(\ell) \mathbf{s}_{i}\right]+u_{n}(\ell)
\end{aligned}
$$

where $u_{n}(\ell)=(1 / M) \mathbf{r}_{n}^{H}\left[v_{0}(\ell) v_{1}(\ell) \cdots v_{M-1}(\ell)\right]^{T}$ is additive noise after beamforming in the $n$th direction and $\eta_{n, k}=(1 / M) \hat{\mathbf{r}}_{n}^{H} \mathbf{r}_{k}$ is the spatial correlation between the $n$th receiver steering vector $\hat{\mathbf{r}}_{n}$ (which in general is $\hat{\mathbf{r}}_{n}=\mathbf{r}_{n} \odot \mathbf{b}$ where $\mathbf{b}$ is a spatial sidelobe taper and $\odot$ is the Hadamard product operator) and the steering vector $\mathbf{r}_{k}$ of the incident radar return signal reflected from the $k$ th mainbeam-illuminated region. The term $\tilde{\mathbf{x}}_{i, \mathrm{SL}, n}(\ell)$ is the superposition of the sidelobe-illuminated range profiles by the the $i$ th radar resulting after beamforming in the $n$th direction at radar 1 . We denote the $i$ th composite range profile 
after beamforming in the $n$th direction at radar 1 as

$$
\tilde{\mathbf{x}}_{i, n}(\ell)=\sum_{k=1}^{K}\left(\eta_{n, k} \mathbf{x}_{i, k}(\ell)\right)+\tilde{\mathbf{x}}_{i, \mathrm{SL}, n}(\ell)
$$

for $i=1,2, \ldots, K$ where $\tilde{\mathbf{x}}_{n, n}(\ell)$ is the range profile of interest and $\tilde{\mathbf{x}}_{i, n}(\ell)$ for $i \neq n$ are the "multistatic interference range profiles." The composite range profile $\tilde{\mathbf{x}}_{i, n}(\ell)$ comprises everything that is illuminated (either mainbeam or sidelobe) by waveform $\mathbf{s}_{i}$, the reflection from which is subsequently received at radar 1 , and then beamformed in the $n$th direction. As such, the $n$ th-beamformer received signal model of (2) can be simplified in terms of the $K$ composite range profiles as

$$
z_{n}(\ell)=\sum_{i=1}^{K} \tilde{\mathbf{x}}_{i, n}^{T}(\ell) \mathbf{s}_{i}+u_{n}(\ell) .
$$

By collecting $N$ time samples of the received radar return signal after beamforming, the resulting signal model from (4) can be expressed in vector notation as

$$
\mathbf{z}_{n}(\ell)=\sum_{i=1}^{K} \tilde{\mathbf{X}}_{i, n}^{T}(\ell) \mathbf{s}_{i}+\mathbf{u}_{n}(\ell)
$$

where $\mathbf{z}_{n}(\ell)=\left[z_{n}(\ell) z_{n}(\ell+1) \cdots z_{n}(\ell+N-1)\right]^{T}$ is $N$ contiguous temporal samples of the received signal after beamforming, $\mathbf{u}_{n}(\ell)=\left[u_{n}(\ell) u_{n}(\ell+1)\right.$ $\left.\cdots u_{n}(\ell+N-1)\right]^{T}$ is a vector of additive noise after beamforming, and $\tilde{\mathbf{X}}_{i, n}(\ell)=\left[\tilde{\mathbf{x}}_{i, n}(\ell) \tilde{\mathbf{x}}_{i, n}(\ell+1)\right.$ $\left.\cdots \tilde{\mathbf{x}}_{i, n}(\ell+N-1)\right]^{T}$ is an $N \times N$ matrix comprised of $N$-length sample-shifted snapshots (in the columns) of the $i$ th composite range profile which results after beamforming in the $n$th direction.

The standard matched filtering operation [5], which is the convolution of the received radar return signal with the time-reversed complex conjugate of the $n$th transmitted waveform, may be applied to the $n$ th-beamformed received signal in (5). The signal flow of multistatic pulse compression using a bank of matched filters is illustrated in Fig. 2. Applying the appropriate matched filter to each of the $K$ beamformed outputs yields the range profile estimate

$$
\hat{x}_{n}(\ell)=\mathbf{s}_{n}^{H} \mathbf{z}_{n}(\ell)
$$

for $n=1,2, \ldots, K$ and $\ell=0,1, \ldots, L-1$. However, since ideal matched filtering assumes only a single received signal in white noise, it is expected (and is shown via simulation in Section V) that the matched filter will perform poorly in the multistatic scenario as the received signals will, in effect, jam one another to the extent that beamforming does not suppress the multistatic interference. Hence, the accurate estimation of one of the $K$ mainbeam-illuminated range profiles necessitates that the corresponding pulse compression

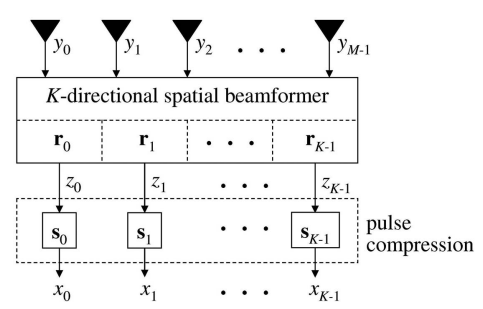

Fig. 2. Multistatic pulse compression signal flow using matched filters.

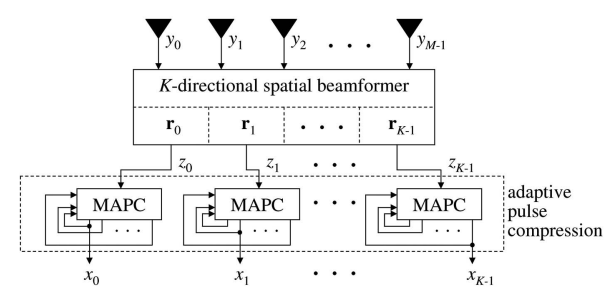

Fig. 3. Multistatic pulse compression signal flow using MAPC.

filter account for the interference associated with each of the other $K-1$ waveforms.

\section{MULTISTATIC ADAPTIVE PULSE COMPRESSION}

To accommodate for multiple, simultaneouslyreceived signals in the same spectrum the MAPC algorithm replaces the single pulse compression matched filter $\mathbf{s}_{n}$ that is applied to the $n$th beamformed output in (6) with a bank of $K$ APC filters that arise from a generalization of the MMSE-based (minimum mean-squared error) APC filter [12-14]. The signal flow of multistatic pulse compression using MAPC is illustrated in Fig. 3 in which, for a given beamformer direction, all of the $K$ composite range profiles are estimated. The estimates of the $K$ composite range profiles are then subsequently employed to adaptively determine the appropriate pulse compression receive filters to use to refine the estimates of the $K$ composite range profiles corresponding to the $n$th receive beamformer at radar 1 . The $i$ th receive filter is found by minimizing the MMSE cost function [17]

$$
J_{i, n}(\ell)=E\left[\left|\tilde{x}_{i, n}(\ell)-\mathbf{w}_{i, n}^{H}(\ell) \mathbf{z}_{n}(\ell)\right|^{2}\right]
$$

with respect to the adaptive filter $\mathbf{w}_{i, n}(\ell)$ for $i=$ $1,2, \ldots, K$ over the composite range cells of interest. The $i$ th MMSE cost function for the $n$th beamformed output (7) is minimized by taking the derivative of (7) with respect to $\mathbf{w}_{i, n}^{*}(\ell)$ and solving which yields

$$
\mathbf{w}_{i, n}(\ell)=\left(E\left[\mathbf{z}_{n}(\ell) \mathbf{z}_{n}^{H}(\ell)\right]\right)^{-1} E\left[\tilde{x}_{i, n}(\ell) \mathbf{z}_{n}(\ell)\right] .
$$

We assume, as in [14], that a range cell's return signal is relatively stationary over the length of the waveform and that neighboring range cells' returns within the same range profile are statistically uncorrelated. Also, we assume that the set of $K$ composite range profiles are independent as well (even if the mainbeam 
footprints overlap, as the aspect angle from which a given region is illuminated can result in substantial variation in the particular realization of the resulting range profile). As a result, and by employing (5), the formulation in (8) simplifies to

$$
\mathbf{w}_{i, n}(\ell)=\rho_{i, n}(\ell)\left(\sum_{k=1}^{K} \mathbf{C}_{k, n}(\ell)+\mathbf{R}_{n}\right)^{-1} \mathbf{s}_{i}
$$

for $i=1,2, \ldots, K$, where $\rho_{i, n}(\ell)=E\left[\left|\tilde{x}_{i, n}(\ell)\right|^{2}\right]$ is the expected power of $\tilde{x}_{i, n}(\ell)$ and $\mathbf{R}_{n}=E\left[\mathbf{u}_{n}(\ell) \mathbf{u}_{n}^{H}(\ell)\right]$ is the temporal (i.e., range) noise covariance matrix after beamforming in the $n$th direction. The matrix $\mathbf{C}_{k, n}(\ell)$ is the $k$ th signal correlation matrix beamformed in the $n$th direction defined as

$$
\mathbf{C}_{k, n}(\ell)=\sum_{\tau=-N+1}^{N-1} \rho_{k, n}(\ell+\tau) \mathbf{s}_{k, \tau} \mathbf{s}_{k, \tau}^{H}
$$

where $\mathbf{s}_{k, \tau}$ contains the elements of the waveform $\mathbf{s}_{k}$ shifted by $\tau$ samples and the remainder zero-filled, e.g. $\mathbf{s}_{k, 2}=\left[\begin{array}{lll}0 & 0 & s_{k}(0) \cdots s_{k}(N-3)\end{array}\right]^{T}$ for $\tau=2$ and $\mathbf{s}_{k,-2}=\left[\begin{array}{llll}s_{k}(2) \cdots s_{k}(N-1) & 0 & 0\end{array}\right]^{T}$ for $\tau=-2$. We observe that (9) is similar to the form found for the monostatic APC algorithm [14]. However, instead of a single received signal, the multistatic formulation in (9) accounts for $K$ superpositioned received signals associated with the $K$ transmitted waveforms. Hence, the resulting filter for the $\ell$ th range cell of the $i$ th composite range profile will attempt to null the interference from other target returns in surrounding range cells within the $i$ th composite range profile as well as interference from target returns in the other $K-1$ composite range profiles.

To employ (9) and (10) requires some knowledge of the $K$ composite range profiles and the noise covariance matrices $\mathbf{R}_{n}$ for $n=1,2, \ldots, K$. Assuming the beamformed noise covariance is white, $\mathbf{R}_{n}$ simplifies to $\sigma_{u}^{2} \mathbf{I}$ in which $\sigma_{u}^{2}$ is the noise power and I is the $N \times N$ identity matrix. Also, while knowledge of the $K$ range profiles is generally not available, an initial estimate can be obtained by applying a matched filter bank. However, as has been noted, the matched filter for a given multistatic waveform does not account for the presence of the other $K-1$ received signals. To that end the MMSE formulation can be employed as in [14] whereby the absence of prior knowledge is represented by initially setting all the range cell estimates of the $K$ composite range profiles to be equal and assuming noise is negligible. It was shown for the monostatic case that this initialization results in an initial MMSE filter that closely resembles the matched filter, which is itself the optimal given no prior knowledge [5]. For the multistatic formulation, (9) reduces to the set of initial MMSE filters

$$
\overline{\mathbf{w}}_{i}=\left(\sum_{k=1}^{K} \overline{\mathbf{C}}_{k}\right)^{-1} \mathbf{s}_{i}
$$

for $i=1,2, \ldots, K$, where the matrix $\overline{\mathbf{C}}_{k}$ is defined as

$$
\overline{\mathbf{C}}_{k}=\sum_{\tau=-N+1}^{N-1} \mathbf{s}_{k, \tau} \mathbf{s}_{k, \tau}^{H} .
$$

The initial MMSE filters from (11) are range invariant and AOA invariant so they can therefore be precomputed for a particular set of transmitted waveforms and applied to each of the $K$ beamformed signals (or a desired subset). After the filters from (11) are applied as in (6) with the matched filter replaced by the set of initial MMSE filters $\overline{\mathbf{w}}_{i}$ for $i=1,2, \ldots, K$ and the $K$ length- $L$ sets of initial composite range profile estimates $\hat{\tilde{x}}_{i, n}(\ell)$ have been obtained, the individual composite range cell power estimates $\hat{\rho}_{i, n}(\ell)=\left|\hat{\tilde{x}}_{i, n}(\ell)\right|^{2}$ for $i=1,2, \ldots, K$ are computed. Subsequently, the composite range cell power estimates $\hat{\rho}_{i, n}(\ell)$ are used in (9) to obtain the adaptive receive filters $\mathbf{w}_{i, n}(\ell)$ which are then reapplied to the beamformed received signal $\mathbf{z}_{n}(\ell)$. This process of refining the adaptive receive filters and then reestimating the sets of composite range cells is repeated for a predetermined number of stages where the estimated composite range cell values for a given stage become prior knowledge for the following stage (which is what fundamentally distinguishes RMMSE estimation [12] from standard MMSE estimation [17]). The adaptive receive filters are able to mitigate the masking effects caused by waveform cross-correlation sidelobes and range sidelobes due to the fact that they are computed based upon the estimated a priori knowledge regarding the large target returns. Hence, as long as sufficient adaptive degrees of freedom are available, the MAPC filters at each successive stage will further improve the estimation accuracy of the $K$ range profiles until reaching the noise floor, at which point little additional improvement is observed.

As with APC in [14], the performance of the MAPC algorithm is determined by the available adaptive degrees of freedom, which is limited by the waveform length $N$ similar to an adaptive sidelobe canceler for array processing applications. However, the presence of $K$ different received signals makes the multistatic shared-spectrum radar problem substantially more difficult than its monostatic counterpart since, on average for a given range cell, the number of targets from which masking interference must be suppressed increases by a factor of $K$ over the monostatic case. When all $K$ radars' mainbeams illuminate different regions, some suppression of the $K-1$ interfering composite range profiles can be expected thereby requiring a smaller portion of the adaptive degrees of freedom for the cancellation of the multistatic interference. Hence, when two or more radars overlap mainbeam illumination regions some degradation is therefore 
expected compared with when sufficient separation of mainbeam regions is available. It is shown in Section V how the MAPC algorithm performs as the number of interference sources increases (i.e., as the number of concurrently operating radars is increased).

\section{IMPLEMENTATION ISSUES}

This section addresses the implementation of the MAPC algorithm with respect to numerical stability and computationally efficiency. While MAPC is applied to a different problem than the original APC algorithm [14], it shares some of the same general structure of APC. Hence, some of the implementation methods used for APC, namely an efficient updating strategy and dynamic range compression to avoid ill-conditioning, can be applied to MAPC as well.

\section{A. Computational Efficiency}

Beyond the initial stage, the RMMSE-based pulse compression techniques necessitate the inversion of an $N \times N$ matrix for every range cell at each stage. For the monostatic APC algorithm presented in [14], a variation of the matrix inversion lemma [18] was employed in which, given $(\mathbf{C}(\ell)+\mathbf{R})^{-1}$ and assuming $\mathbf{R}$ has the form of a diagonal matrix scaled by the noise power $\sigma_{u}^{2}$, a rank-3 update is applied to yield $(\mathbf{C}(\ell+1)+\mathbf{R})^{-1}$. Therefore, the monostatic APC only requires a single matrix inverse at each stage.

This approach can, in general, be applied for the MAPC algorithm as well. For MAPC, we note that for the $n$th beamformer direction, the matrix $\left(\sum_{k=1}^{K} \mathbf{C}_{k, n}(\ell)+\mathbf{R}_{n}\right)$ is the same for all $K$ filters $\mathbf{w}_{i, n}$. Hence, a single rank-3 update is required per range cell for the $K$ composite range profiles comprising the $n$th beamformed signal. Also, the processing involved for each beamformed signal is independent of the processing (if any) for the other beamformed signals and as such readily enables parallel implementation of MAPC for each individual beamformed signal.

Even with the significant reduction in computational complexity afforded by the efficient updating strategy, the implementation of MAPC may not be feasible for real-time operation in current radar systems. However, many applications of radar whereby a multistatic configuration has been envisioned (such as a cluster of space-based radars) do not necessarily require real-time operation as much of the data may be processed off-line at a later time. Further research is ongoing to develop faster and more efficient implementation strategies for MAPC.

\section{B. Numerical Stability}

The matrix $\left(\sum_{k=1}^{K} \mathbf{C}_{k, n}(\ell)+\mathbf{R}_{n}\right)$ could potentially become ill-conditioned in the vicinity of very large targets or when small range cell estimates approach zero. However, a heuristic approach similar to that described in [14] will work for the multistatic case which is to replace $\hat{\rho}_{i, n}(\ell)=\left|\hat{\tilde{x}}_{i, n}(\ell)\right|^{2}$ with $\hat{\rho}_{i, n}(\ell)=$ $\left|\hat{\tilde{x}}_{i, n}(\ell)\right|^{\alpha}$ and (under the white noise assumption) replace the noise power $\sigma_{u}^{2}$ used to estimate the MMSE adaptive filters with $\sigma_{u}^{\alpha}$, for $0 \leq \alpha \leq 2$. For the case of large SNR scatterers, using $\alpha<2$ reduces the effective SNR dynamic range and thereby alleviates the possibility of ill-conditioning. It has been found based upon extensive experimentation via simulation that values of $1.1 \leq \alpha \leq 1.7$ with 2 to 4 stages of the MAPC algorithm (excluding the initialization stage) tend to yield the best results. Furthermore, $\alpha$ should be set at the high end (near 1.7) initially to quickly drive down the sidelobes from large SNR scatterers and then decrease (to near 1.1 at the final stage). For initialization using (11) and (12), $\alpha$ can be set to 2. It is a topic of future research to determine if optimal values of $\alpha$ can be found as a function of the surrounding composite range cell estimates.

An additional heuristic approach that can be used to alleviate possible ill-conditioning is to set a lower bound upon the magnitudes of the composite range cell estimates. This is done so that slightly larger values of $\alpha$ can be used to drive down the sidelobes from large scatterers more quickly without driving smaller range cell estimates to zero.

\section{SIMULATION RESULTS}

To demonstrate the performance of the MAPC algorithm we compare its performance with that of matched filtering and least-squares estimation in the multistatic shared-spectrum environment. For all cases, without loss of generality, we determine the performance of the three techniques at radar 1 with the receive beam steered to the AOA of the return signal from the mainbeam illumination of radar 1 (the same processing is performed at each particular radar receiver and for each receiver beamformed signal). In other words, the composite range profile of interest is $\tilde{x}_{1,1}(\ell)$ while the interference composite range profiles that must be suppressed are $\tilde{x}_{k, 1}(\ell)$ for $k=2, \ldots, K$. The first and second cases we examine involve $K=2$ radars each illuminating a completely different mainbeam range profile with the respective composite range profiles each consisting of a single large target and a single small target. The first case involves no Doppler mismatch while for the second case the large target in each respective composite range profile induces a relatively large Doppler shift thereby resulting in Doppler mismatch (it has been found for monostatic APC processing that Doppler mismatch on the largest target causes the most significant reduction in performance). The third case also involves $K=2$ radars with each composite 
range profile being target rich with some of the targets being Doppler mismatched. We also examine an even more stressing scenario with $K=4$ radars in which the four composite range profiles are target-rich and contain targets with Doppler. Finally, the mean-square error (MSE) performance is determined for the estimation of the mainbeam-illuminated composite range profile of radar 1 as a function of the number of simultaneously operating radars. In all cases randomly generated polyphase waveforms of length $N=30$ are employed that possess typical auto- and cross-correlation properties (i.e., not optimized for any particular criteria).

Each of the $K$ ground truth radar range profiles consists of targets of highly varying power levels. Additive noise is modeled as zero-mean complex Gaussian with a power level of $-60 \mathrm{~dB}$ relative to the largest target's power level. As a result of transmitter and/or receiver sidelobes, the $K-1$ interference signals will be suppressed somewhat relative to the mainbeam signal of interest. While the relative received power levels at a given receiver are dependent upon the respective transmit powers, the distances from the illuminated regions to the particular receiver, and the spatial AOAs, for simplicity we model the largest target in each of the composite range profiles as being $20 \mathrm{~dB}$ below that of the largest target in the composite range profile of interest. As such, while the composite range profile of interest $\tilde{x}_{1,1}(\ell)$ dominates the return signal, the multistatic interference substantially limits the sensitivity with which $\tilde{x}_{1,1}(\ell)$ can be estimated. Also, note that while the noise power appears quite low, it is relative to the largest target return in $\tilde{x}_{1,1}(\ell)$ and the resulting SNR for the small targets is representative of what one could typically expect in practice. For all cases the processing windows of the ground truth range profiles consist of $L=100$ range cells that correspond to concurrently received portions of the $K$ individual radar return signals. Also, for MAPC a lower bound is set on the range cell estimate magnitudes which prevents the range cell estimate values from decreasing much below the normalized noise floor.

In the examples to be shown we show results for all of the composite range profiles. Note that only composite range profile 1 is sent to the detector after MAPC processing. All other composite range profiles are used only to estimate $\tilde{x}_{1,1}(\ell)$.

\section{A. Two Multistatic Radars Operating in Sparse Target Environment}

For the first case, we consider $K=2$ radars concurrently operating in the same spectrum. For these examples we make the simplifying assumption that the direct path illumination (i.e., without reflection) from one radar to another can be effectively blanked. Each radar has an $M=11$
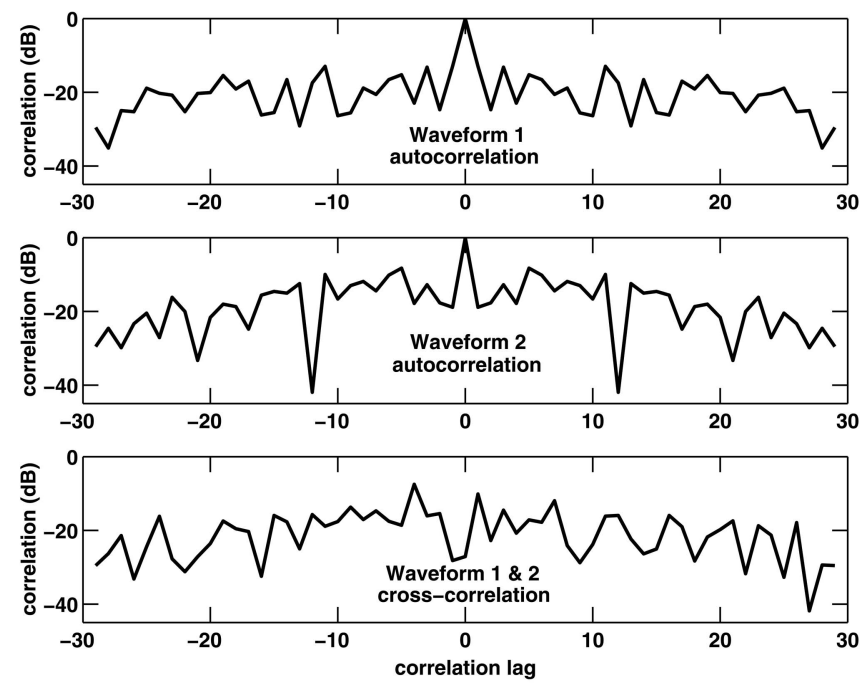

Fig. 4. Correlations of transmitted waveforms.

element uniform linear array. Occupying the same spectrum, each radar concurrently transmits a different waveform, the respective auto-correlations and cross-correlation of which are depicted in Fig. 4. We examine the performance of the standard matched filter, LS estimator [9-11], and the MAPC algorithm when applied to estimate the mainbeam range profile illuminated by radar 1 at the receiver of radar 1 . Note that unlike MAPC, the matched filter and least-squares estimator are only appropriate for the extraction of monostatic radar return signals and are thus expected to perform poorly for the multistatic radar scenario. The independent composite range profiles each contain a (different) large target and a (different) nearby small target that would otherwise be masked by the matched filter range sidelobes in the standard monostatic scenario. Four stages are employed for the MAPC algorithm (inclusive of the initial stage employing (11)) with the robustness parameter $\alpha$ set as $2,1.7,1.4$, and 1.3 for the four stages, respectively.

The results for this scenario are illustrated in Fig. 5, in which the black line represents the ground truth of the respective composite range profiles and the top and bottom plot in the figure pertain to the composite range profile of interest $\tilde{\mathbf{x}}_{1,1}(\ell)$ and the interfering composite range profile $\tilde{\mathbf{x}}_{2,1}(\ell)$ from the perspective of radar 1, respectively. For illustrative purposes, we apply the matched filter and least-squares estimator to the received beamformed signal corresponding to each of the $K$ waveforms (this would not otherwise be done as neither of these approaches make use of this multistatic interference information). As expected, the matched filter estimate of $\tilde{x}_{1,1}(\ell)$ is rather poor due to the combination of range sidelobes and mutual interference resulting from the presence of more than one radar return signal yielding an MSE for composite range profile 1 of $-19 \mathrm{~dB}$. The least-squares estimator performs considerably better than the matched filter with an 

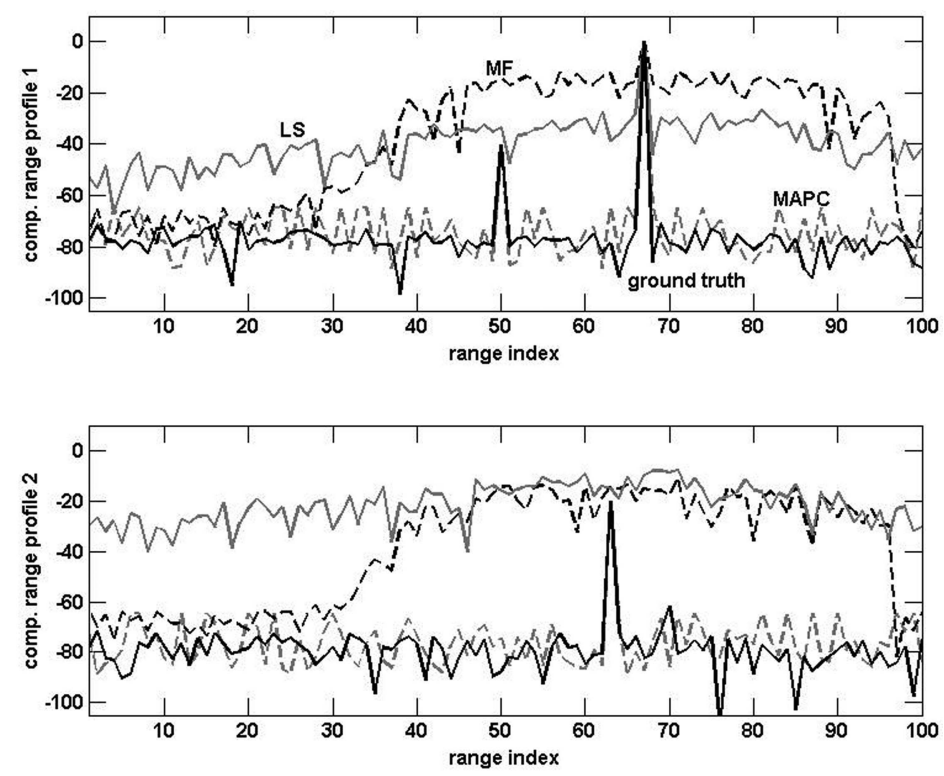

Fig. 5. Sparse target scenarios without Doppler mismatch, (upper) desired composite range profile $\tilde{\mathbf{x}}_{1,1}$, (lower) interference composite range profile $\tilde{\mathbf{x}}_{2,1}$.
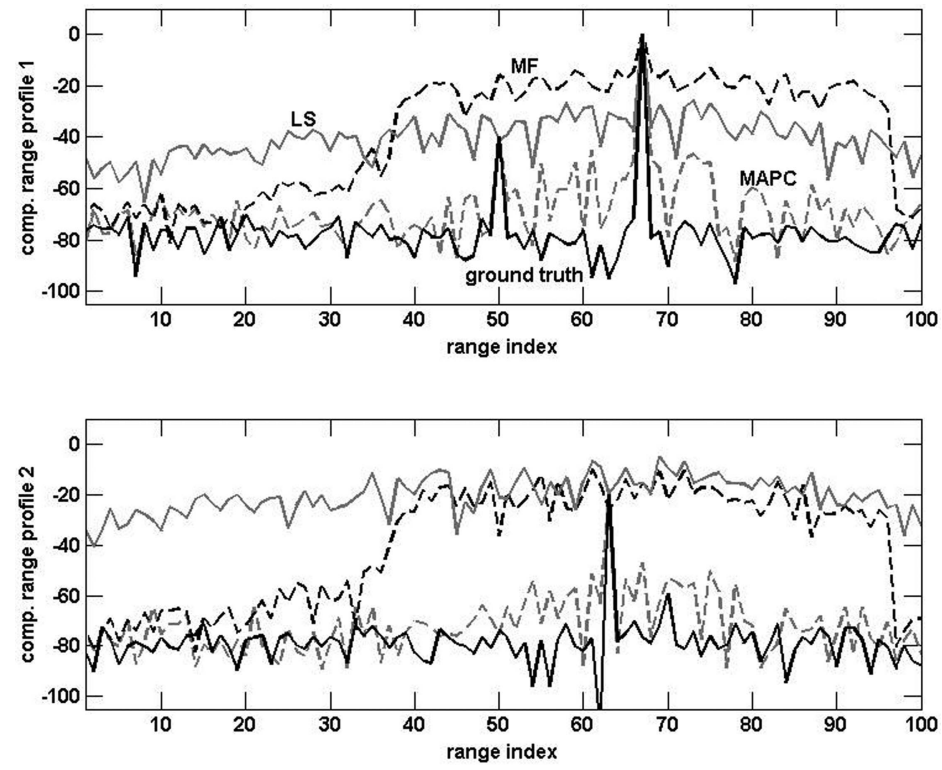

Fig. 6. Sparse target scenarios with Doppler mismatch, (upper) desired composite range profile $\tilde{\mathbf{x}}_{1,1}$, (lower) interference composite range profile $\tilde{\mathbf{x}}_{2,1}$.

MSE of $-35 \mathrm{~dB}$. However, the MAPC approach substantially outperforms both matched filtering and least-squares estimation because it accounts for the presence of both received return signals and thereby is able to suppress the range sidelobes as well as the mutual interference achieving, over the four stages, successive MSE levels for composite range profile 1 of $-23 \mathrm{~dB},-45 \mathrm{~dB},-67 \mathrm{~dB}$, and $-70 \mathrm{~dB}$, respectively. Furthermore, only the MAPC approach is able to uncover the small target in composite range profile 1 .

To examine the effects of Doppler upon the MAPC algorithm, Doppler shifts of $+4^{\circ}$ and $-6^{\circ}$ over the lengths of the respective waveforms exist for the large targets in composite range profiles $\tilde{x}_{1,1}(\ell)$ and $\tilde{x}_{2,1}(\ell)$, respectively (e.g. a $6^{\circ}$ Doppler shift would result for a Mach 2 target illuminated by a $1 \mu \mathrm{s}$ S-band pulse similar to FAA air traffic control radars). The operation of the MAPC algorithm is performed as before. Fig. 6 demonstrates that the MAPC algorithm experiences a marked increase in the sidelobe levels with the overall MSE for MAPC found to be $-24 \mathrm{~dB}$, $-43 \mathrm{~dB},-47 \mathrm{~dB}$, and $-47 \mathrm{~dB}$ for the four stages.

However, MAPC is Doppler tolerant enough that the targets remain visible above the sidelobes and noise. Compared with the matched filter, which attains an 

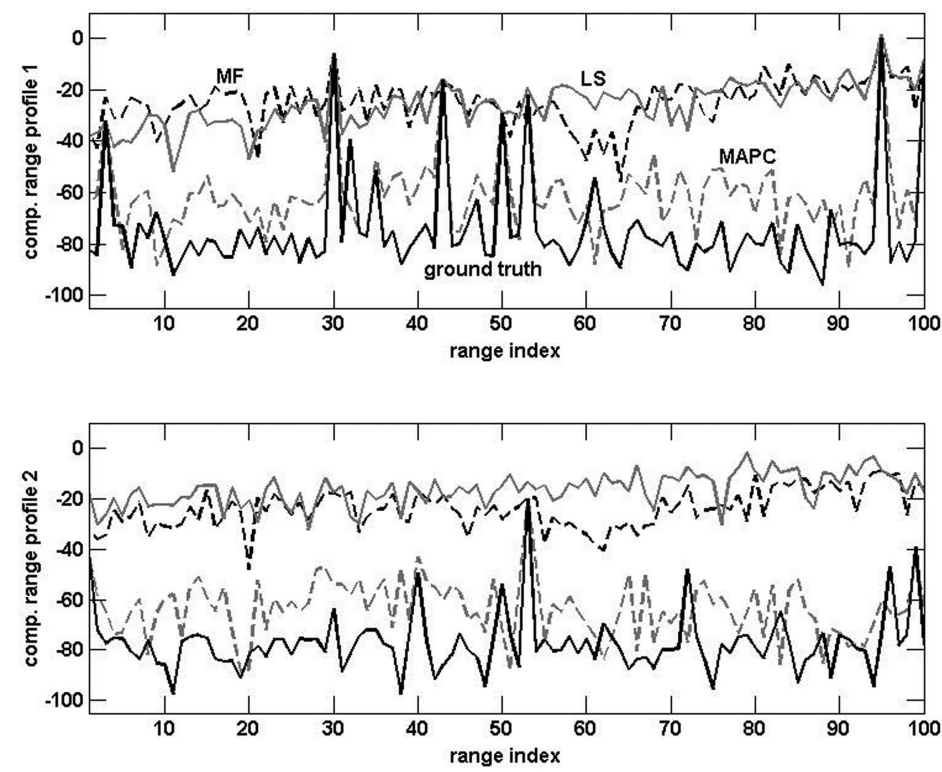

Fig. 7. Dense target scenarios with Doppler mismatch, (upper) desired composite range profile $\tilde{\mathbf{x}}_{1,1}$, (lower) interference composite range profile $\tilde{\mathbf{x}}_{2,1}$.

MSE of $-21 \mathrm{~dB}$, and least-squares, which reaches an MSE of $-35 \mathrm{~dB}$, the MAPC algorithm is still found to provide a significant improvement.

\section{B. Two Multistatic Radars Operating in Dense Target Environment}

For the dense target scenario involving $K=2$ radars, we examine the estimation of $\tilde{x}_{1,1}(\ell)$ at radar 1 in which targets of various power levels are distributed among the two composite range profiles (we again model the largest target in $\tilde{x}_{1,2}(\ell)$ as being $20 \mathrm{~dB}$ below the largest target in $\tilde{x}_{1,1}(\ell)$. The targets illuminated by both radars have Doppler shifts over the length of the waveform that are randomly assigned from a uniform distribution on $\left[-3^{\circ}, 3^{\circ}\right]$. As before, four stages are employed for MAPC.

Fig. 7 shows the performance for the dense target scenario in which the matched filter and least-squares estimator attain an MSE for composite range profile 1 of $-20 \mathrm{~dB}$ and $-21 \mathrm{~dB}$, respectively. The decoupling capability of the MAPC algorithm, however, is found to achieve overall MSE levels of $-23 \mathrm{~dB}$, $-36 \mathrm{~dB},-44 \mathrm{~dB}$, and $-52 \mathrm{~dB}$ over the four stages, respectively. In so doing, MAPC enables the detection of many of the targets that are otherwise completely masked by range sidelobes and mutual interference when using either the matched filter or least-squares estimator.

\section{Four Multistatic Radars Operating in Dense Target Environment}

For an even more stressing environment, we examine the performance of the MAPC algorithm with $K=4$ radars concurrently operating in the same spectrum. The four waveforms have similar auto- and cross-correlation properties as those in Fig. 4. The composite range profile associated with each of the four waveforms contains targets with Doppler shifts over the length of the waveform that are randomly assigned from a uniform distribution on $\left[-3^{\circ}, 3^{\circ}\right]$. Fig. 8 illustrates the performance comparison where it is clear that the MAPC algorithm outperforms significantly the standard matched filter and least-squares estimator. The MSE for composite range profile 1 is found to be $-21 \mathrm{~dB}$ for the matched filter and $-27 \mathrm{~dB}$ for least-squares. By comparison, the MAPC algorithm achieves $-20 \mathrm{~dB},-31 \mathrm{~dB}$, $-40 \mathrm{~dB}$, and $-45 \mathrm{~dB}$ for the four successive stages, respectively, and again enables the detection of targets that would have otherwise been masked.

\section{MSE Performance as Function of the Number of Radars}

Finally, observe that the sidelobe levels and resulting MSE for MAPC in the $K=4$ case are higher than that for the similar $K=2$ case. This is due to the increased number of interference sources. In order to quantify the performance of MAPC as a function of the number of interference sources we determine the MSE performance according to the numbers of concurrently operating radars in the same spectrum. Each radar employs a length $N=30$ randomly generated polyphase waveform and the set of $K$ composite range profiles from the perspective of radar 1 each contain two different randomly distributed targets, one target being $40 \mathrm{~dB}$ below the other. The targets possess no radial motion and thus there are no Doppler mismatch effects. The number of operating radars is varied from $K=1$ to 30 with 100 independent trials performed for each. Two different 

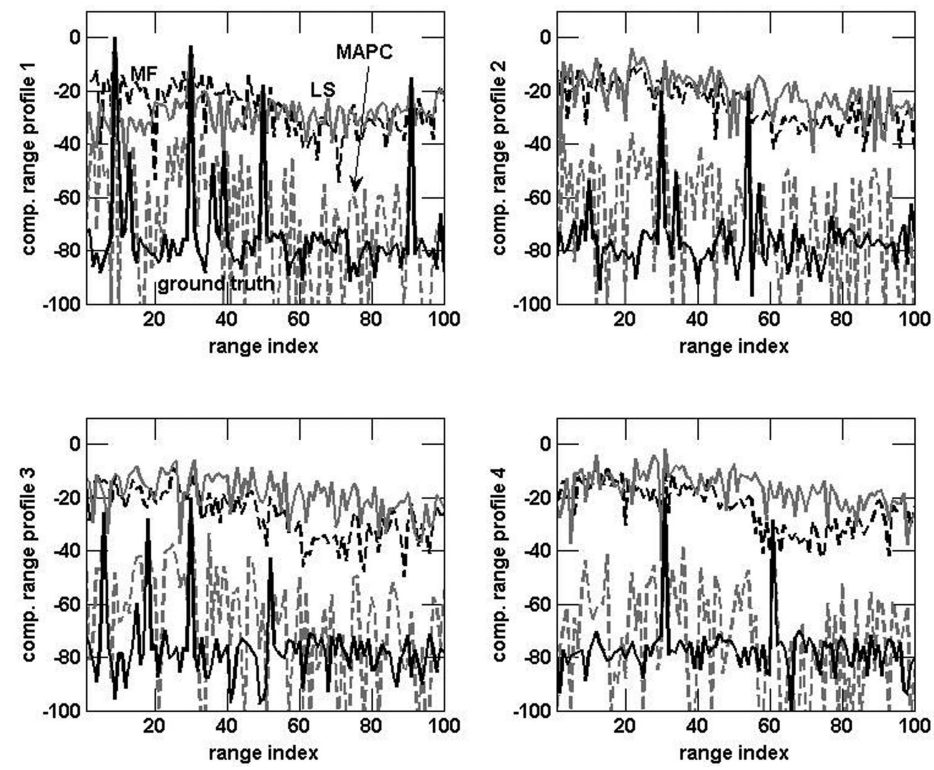

Fig. 8. Dense target scenarios with Doppler for four multistatic radars, (upper-left) $\tilde{\mathbf{x}}_{1,1}$, (upper-right) $\tilde{\mathbf{x}}_{2,1}$, (lower-left) $\tilde{\mathbf{x}}_{3,1}$, (lower-right) $\tilde{\mathbf{x}}_{4,1}$.

scenarios are considered. In the first scenario all of the the interference composite range profiles (i.e., $\tilde{x}_{k, 1}(\ell)$ for $k=2, \ldots, K)$ are received through transmitter and/or receiver sidelobes and are thus assumed to be suppressed by $20 \mathrm{~dB}$ with respect to the range profile of interest $\tilde{x}_{1,1}(\ell)$. In the second scenario we examine the performance when the $K$ radars all illuminate the same region and such that the interference composite range profiles are received at the same power as the composite range profile of interest. Both of these scenarios are, obviously, contrived cases yet they aptly illustrate the performance of MAPC as a function of the number and level of the multistatic interference present.

The resulting MSE curves for the estimation of composite range profile 1 by MAPC with 4 stages as before and by the matched filter for both scenarios are depicted in Fig. 9. The matched filter is found to achieve at most $-20 \mathrm{~dB}$. When transmit/receive beamforming is not capable of suppressing the portion of the received signal associated with the other $K-1$ waveforms the matched filter performance is found to degrade significantly reaching $-5 \mathrm{~dB}$ for the case when $K=30$ radars are present. By comparison, the MAPC algorithm achieves $-70 \mathrm{~dB}$ MSE for a single radar and increases to at most $-32 \mathrm{~dB}$ for 30 radars with mainbeams illuminating different regions (thus enabling transmit/receive beamformer gain). When the $K$ radars all illuminate the same region no transmit/receive beamformer interference suppression is possible and as such degradation in MAPC performance is expected as was found for the matched filter. However, it is found that the MSE for composite range profile 1 is roughly equal or even slightly lower for this case (at least for low values of $K$ ) relative to when spatial suppression is available.

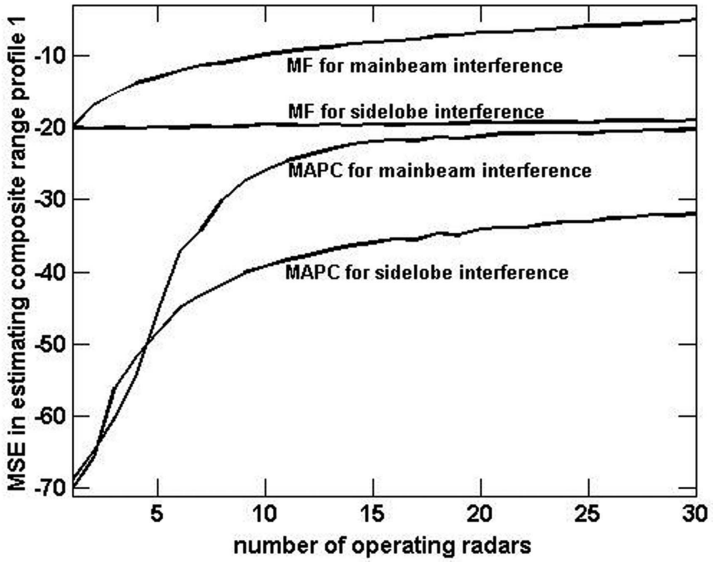

Fig. 9. MSE as function of number of simultaneously operating radars.

This effect is likely due to the fact that a higher power interference signal can be more accurately estimated and thus more adequately suppressed by the range nulling of MAPC. However, as the value of $K$ increases we see that, as expected, the MSE for this case does become larger than the previous case as more of the adaptive degrees of freedom are used to suppress the higher levels of interference. At $K=30$ radars the MAPC algorithm achieves an MSE of $-20 \mathrm{~dB}$ which, by the way, is the same as was found for the matched filter for a single radar. In both cases it is found that MAPC degrades gracefully as the number of interference sources increases.

\section{DISCUSSION AND SUMMARY}

Previously, shared-spectrum multistatic radar has not been considered feasible due to the cross-correlations of the multistatic waveforms which 
may produce ambiguities that manifest in the form of higher sidelobes. In this work a technique denoted as MAPC has been introduced whereby multiple received radar return signals are jointly pulse compressed in order to suppress both range and cross-correlation sidelobes. The MAPC algorithm is a generalization of the monostatic APC algorithm which has previously been shown via simulation to suppress even very large range sidelobes into the noise thereby greatly increasing the sensitivity of the radar. The MAPC algorithm has been demonstrated via simulation to suppress both range sidelobes and cross-correlation sidelobes into the noise for the shared-spectrum multistatic radar configuration while both standard matched filtering and least-squares estimation are found to perform rather poorly by comparison as they are essentially monostatic techniques. Whereas the matched filter maximizes the SNR of a point target in white noise, the MAPC algorithm adaptively determines the appropriate receive filter to employ for each individual range cell of each received radar return signal such that the masking sidelobes from larger proximate targets are mitigated with minimal mismatch loss in the MSE sense. In a shared-spectrum multistatic environment with several relatively fast-moving targets of various SNRs and the radar mainbeams illuminating different regions, the MAPC algorithm is found to yield, for the particular range profile of interest, an MSE improvement of $28 \mathrm{~dB}$ and $13 \mathrm{~dB}$ over matched filtering and least-squares for $K=2$ radars, respectively, and an MSE improvement of $23 \mathrm{~dB}$ and $20 \mathrm{~dB}$ over matched filtering and least-squares for $K=4$ radars, respectively. Furthermore, the performance of MAPC has been explored as a function of the number of concurrently operating shared-spectrum radars and has been shown via simulation to provide MSE improvement over matched filtering of as much as $25 \mathrm{~dB}$ for 6 radars with the MSE performance of MAPC degrading gracefully as the number of concurrently operating radars in the same spectrum increases.

\section{REFERENCES}

[1] Cohen, L. S. Radar spectrum management issues and problems. In Proceedings of the Tri-Service Radar Symposium, Albuquerque, NM, June 21-25, 2004.

[2] Baker, C. J., and Hume, A. L.

Netted radar sensing. IEEE Aerospace and Electronic Systems Magazine, 18, 2 (Feb. 2003), 3-6.

[3] Purdy, D. S.

Receiver antenna scan rate requirements needed to implement pulse chasing in a bistatic radar receiver. IEEE Transactions on Aerospace and Electronic Systems, 37, 1 (Jan. 2001), 285-288.
[4] Verdu, S.

Multiuser Detection.

New York: Cambridge University Press, 1998.

[5] Skolnik, M. I.

Introduction to Radar Systems (3rd ed.).

New York: McGraw-Hill, 2001, 339-369.

[6] Goodman, N. A., Lin, S. C., Rajakrishna, D., and Stiles, J. M.

Processing of multiple-receiver spaceborne arrays for wide-area SAR.

IEEE Transactions on Geoscience and Remote Sensing, 40, 4 (Apr. 2002), 841-852.

[7] Kreiger, G., Fiedler, H., Mittermayer, J., Papathanassiou, K., and Moreira, A.

Analysis of multistatic configurations for spaceborne SAR interferometry.

IEE Proceedings, Pt. F, 150, 3 (June 2003), 87-96.

[8] Cerutti-Maori, D. J. E., and Ender, J. H. G.

An approach to multistatic spaceborne SAR/MTI processing and performance analysis.

IEEE IGARSS, July 21-25, 2003, 4446-4449.

[9] Ackroyd, M. H., and Ghani, F.

Optimum mismatched filter for sidelobe suppression. IEEE Transactions on Aerospace and Electronic Systems, AES-9 (Mar. 1973), 214-218.

[10] Felhauer, T.

Digital signal processing for optimum wideband channel estimation in the presence of noise.

IEE Proceedings, Pt. F, 140, 3 (June 1993), 179-186.

[11] Song, S. M., Kim, W. M., Park, D., and Kim, Y.

Estimation theoretic approach for radar pulse compression processing and its optimal codes.

Electronic Letters, 36, 3 (Feb. 2000), 250-252.

[12] Blunt, S. D., and Gerlach, K.

A novel pulse compression scheme based on minimum mean-square error reiteration.

In Proceedings of the IEEE International Radar

Conference, Sept. 3-5, 2003, 349-353.

[13] Blunt, S. D., and Gerlach, K.

Adaptive pulse compression.

In Proceedings of the IEEE National Radar Conference, Apr. 26-29, 2004, 271-276.

[14] Blunt, S. D., and Gerlach, K.

Adaptive pulse compression via MMSE estimation. IEEE Transactions on Aerospace and Electronic Systems, 42, 2 (Apr. 2006), 572-584.

[15] Blunt, S. D., and Gerlach, K.

Joint adaptive pulse compression to enable multistatic radar.

Presented at the IEE Waveform Diversity and Design Conference, Edinburgh, Scotland, Nov. 8-10, 2004.

[16] Blunt, S. D., and Gerlach, K.

Aspects of multistatic adaptive pulse compression. In Proceedings of the IEEE National Radar Conference, May 9-12, 2005, 104-108.

[17] Kay, S. M.

Fundamentals of Statistical Signal Processing: Estimation Theory.

Upper Saddle River, NJ: Prentice-Hall, 1993, 219-286 and 344-350.

[18] Moon, T. K., and Stirling, W. C.

Mathematical Methods and Algorithms for Signal

Processing.

Upper Saddle River, NJ: Prentice Hall, 2000, 258-264. 


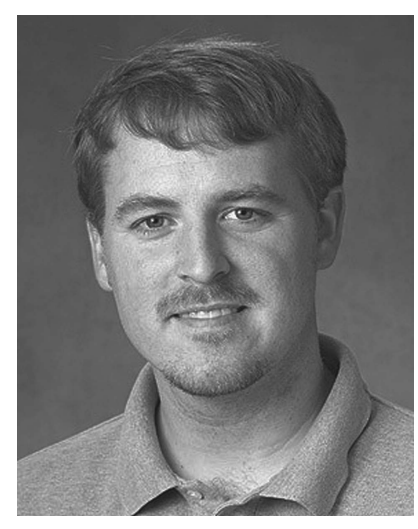

Shannon D. Blunt (S'96-M'02) received the B.S., M.S., and Ph.D. degrees in electrical engineering in 1999, 2000, and 2002, respectively, from the University of Missouri-Columbia (MU).

From 2002 to 2005 he was with the Radar Division of the U.S. Naval Research Laboratory in Washington, D.C. In 2005 he joined the faculty of the Department of Electrical Engineering and Computer Science at the University of Kansas.

Dr. Blunt received the Donald K. Anderson Graduate Student Teaching award in electrical engineering from MU in 2000 and the MU Outstanding Graduate Student award in electrical engineering in 2001. He also received the 2004 Naval Research Laboratory Alan Berman Research Publication award. He is a member of Eta Kappa Nu and Tau Beta Pi. His research interests are in adaptive signal processing for radar and communications with an emphasis on waveform diversity techniques.

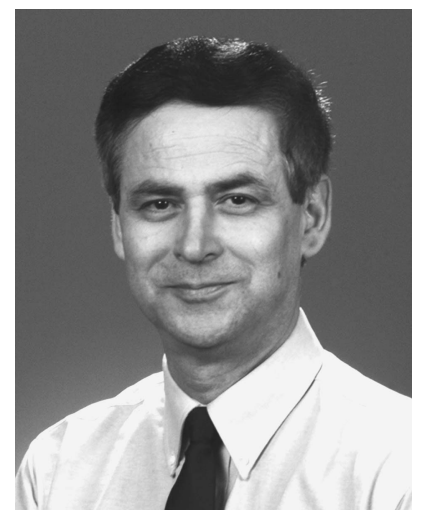

Karl Gerlach (M'81-F'02) was born in Chicago, IL. He received his B.S. in 1972 from the University of Illinois, Urbana, and his M.S. and D.Sc. from George Washington University, Washington, D.C., in 1975 and 1981, respectively, all in electrical engineering.

Since 1972, he has been employed by the Naval Research Laboratory in Washington, D.C. From 1972 to 1976, he worked on experimental submarine communications systems and from 1976 to the present he has been with the Radar Division where his research interests include adaptive signal processing and space-based radar.

Dr. Gerlach was the 1986 recipient of the IEEE AESS Radar Systems Panel award. 